Editorial

\title{
Comparative study of the outcome of pediatric subacute sclerosing pan-encephalitis patients treated with oral ribavirin versus intraventricular interferon alpha $2 \mathrm{~b}$ via Ommaya reservoir
}

\author{
Ghosh K.C. ${ }^{1}$, Bhattacharya R. ${ }^{2}$, Ghosh S. ${ }^{3}$, Mahata M. ${ }^{4}$, Das S. ${ }^{5}$, Das S. ${ }^{6}$, Mondal G.P. ${ }^{7}$ \\ ${ }^{1}$ Dr. Kartick Chandra Ghosh, Associate Professor, ${ }^{2}$ Dr. Ramesh Bhattacharya, Associate Professor, ${ }^{3}$ Dr. Saikat Ghosh, \\ Resident, ${ }^{4}$ Dr. Manoj Mahata, Resident, ${ }^{5}$ Dr. Sarbajit Das, Resident, ${ }^{6}$ Dr. Suman Das, Resident, ${ }^{7}$ Dr. Gourango Prosad \\ Mondal, Professor, all authors are affiliated with Department of Neurology, Calcutta National Medical College, Kolkata, \\ West Bengal, India.
}

Corresponding Author: Dr. Suman Das, Post Doctoral Resident, Department of Neurology, Calcutta National Medical College, 24, Gorachand Road, Kolkata, India. Email: dr.sumands@gmail.com

\begin{abstract}
Introduction: Subacute sclerosing pan-encephalitis is a childhood disease occurring after a latent period of 2-12 years due to persistent defective measles virus within the neurons and glial cells. Various drugs have been used in treating SSPE with mixed success. Methods: All patients diagnosed with SSPE at the Neurology department from January 2011 to March 2018 were included in the study. Initially (upto January 2015) all patients of SSPE were treated with oral ribavirin (Group 1, N=8). Thereafter, when we received the Government supply of interferon alpha $2 b$, we shifted to its intraventricular administration via Ommaya reservoir (Group 2, N=9). The data was retrospectively collected from the medical records of the patients. Results: No statistically significant difference existed in the baseline parameters of the patients of the two groups. Patients of Group 2 had significantly better outcome in terms of reduction in Jabbour staging, cerebrospinal fluid measles antibody titre and Brief Assessment Examination scores. Lower age, lower latent period between measles infection and onset of clinical features of SSPE and higher Jabbour staging predicted the poor outcome in the univariate analysis. Conclusion: Intraventricular interferon alpha therapy is far superior to oral ribavirin. However, all studies with interferon alpha did not report significantly favorable outcome, thereby, necessitating further multicentric studies with larger sample sizes.
\end{abstract}

Key words: Subacute sclerosing pan-encephalitis, Interferon alpha-2, Ribavirin, Intraventricular injection.

\section{Introduction}

Subacute sclerosing pan-encephalitis occurs due to persistent defective measles virus within the neurons and glial cells. Aberrant mutated matrix and envelope protein interfere with assembly and budding of the virions, which therefore, remain intracellular and spread by cell to cell contact [1].

The exact factors that influence viral persistence are unclear, but several immunological factors including low cerebrospinal fluid interferon levels have been implicated. Saha [2] reported an annual incidence of 21 per million population in India. Most patients with SSPE have a history of primary measles infection at $<2$ years, which is followed, after a latent period of 2-12

Manuscript received: $8^{\text {th }}$ June 2018

Reviewed: $18^{\text {th }}$ June 2018

Author Corrected: $26^{\text {th }}$ June 2018

Accepted for Publication: $30^{\text {th }}$ June 2018 years, by the onset of progressive neurological disorder, hence, SSPE is commonly a disease of childhood [1,3]. Children infected with measles $\leq 1$ year of age are 16 times more prone to develop SSPE than those infected at $\geq 5$ years [4]. Although primary measles infection shows no sex disparity, male preponderance has been seen for SSPE (male/female ratio 3:1). The incidence is higher among rural children, especially those living in overcrowded environments, children with two or more siblings, with a lower birth order, with mental retardation and unimmunized with measles vaccine. A close temporal relationship of measles with other viral (Epstein-Barr or parainfluenza type-1) infection modify the course of acute measles infection and may predispose to the development of SSPE [5]. The disease is characterized by cognitive decline, deterioration of motor function and epilepsy, which may be punctuated by periods of clinical plateau, but a gradual progressive 
Editorial

course leading to death, is invariable [1,3,5]. Several disease modifying drugs have been used in the treatment of SSPE- interferon alpha (via intraventricular and intrathecal routes), subcutaneous interferon beta, isoprinosine, intravenous immunoglobulin, amantadine, corticosteroids, acyclovir and ribavirin. Isoprinosine and interferon $\alpha$, have received the most attention [6] Isoprinosine is an immunomodulator that facilitates lymphocyte immune function by promoting lymphocyte proliferation and immunoglobulin and lymphokines production, thereby, improving the general immunodepressive state triggered by a viral infection. Interferon has received attention because it activates natural killer cells and directly inhibits viral replication. Studies have shown that interferon alpha levels are low in patients with SSPE and in vitro studies of patient-derived peripheral mononuclear cells fail to produce interferon alpha in response to stimulation. Thus, exogenous interferon alpha may potentiate the activity of NK cells and promote clearance of the virus $[6,7]$.

This retrospective study aims to compare the outcomes of 2 groups of SSPE patients following administration of oral ribavirin (Group1) and intraventricular interferon alpha (Group 2).

\section{Methods}

Place of study- Neurology Department, Calcutta National Medical College.

Inclusion criteria and sampling technique- All patients diagnosed with SSPE from January 2011 to March 2018 were included in the study by consecutive sampling technique (it is a total enumerative sampling technique in which every subject meeting the criteria of inclusion is selected). Patients were diagnosed with SSPE if they fulfilled 3 out of the following criteria [8] - 1) Clinical- progressive, subacute metal deterioration with typical signs like myoclonus, 2) Electroencephalography (EEG)- periodic, stereotyped, synchronous, high voltage (200-500 mv) bursts of polyphasic, delta waves, being identical in any given lead and repeating at fairly regular (4-10 second) intervals and having 1:1 relationship with myoclonic jerks, 3) cerebrospinal fluid (CSF)- raised gammaglobulin or oligoclonal pattern, 4) Measels antibody- raised titre in serum $(\geq 1: 256)$ and/or cerebrospinal fluid $(\geq 1: 4)$. Jabbour staging of the disease [9], complete blood counts, liver and renal function tests, CSF study for other viral serologies (herpes simplex and Japanese encephalitis viruses), and human immunodeficiency virus (HIV) serology were done for every patient.
Method of study- Initially (upto January 2015) all patients of SSPE were treated with oral ribavirin (15 $\mathrm{mg} / \mathrm{kg} /$ day in two divided doses for 6 weeks) [Ribavin Syrup/tablets, Lupin Laboratories, Pinnacle Division], because those patients from the financially weaker section of the society could not afford interferon alpha and isoprinosine and intravenous formulation of ribavirin were not commercially available. However, with the supply of interferon alpha being provided by the Department of Health and Family Welfare, Government of West Bengal, beyond January 2015, we switched our treatment plan of SSPE patients to interferon alpha- 2b. All patients diagnosed with SSPE had an Ommaya reservoir implanted under the scalp and one catheter was inserted into the lateral ventricle. Intraventricular recombinant interferon alpha-2b (3 million units) [Intalfa 3 MU, Intas Pharmaceuticals Ltd, India] was administered through this reservoir twice weekly for 6 weeks. Mild thrombocytopenia and fevers with injections occurred but did not interfere with the treatment. All patients also received one or more anticonvulsants like sodium valproate, levetiracetam, zonisamide and clonzepam and other supportive therapy (antibiotics for intercurrent infections, trihexyphenidyl for dystonia). After completion of 6 weeks therapy, repeat CSF examination was done to assess the measles antibody titre. The outcome measures were improvement in Brief Assessment Examination (BAE) scores [10], and reduction in the antibody titres in CSF.

In BAE, 1) alertness, 2) language and cognitive function, 3) visual digit span, 4) mathematics, 5) speech and 6) pictoral memory are tested and points ranging from $0-8$ are assigned. Total Score is out of 60 with greater scores reflecting greater impairment. Modifications are outlined for children less than 6 years of age. These patients were followed up on outpatient basis after discharge.

Type of study- Retrospective study. The data of the patients (clinico-epidemiological profile, laboratory reports, and outcome parameters after 6 weeks of treatment) were collected from the medical records.

Statistical analysis- Descriptive statistics were used to describe patient characteristics. Continuous variables were tested on normality and presented as mean and standard deviation [SD]. Univariate logistic-regression analyses were used to estimate the unadjusted odds ratios (OR) and the corresponding 95\% confidence intervals (CI). All tests were 2 -sided, and $P<0.05$ was considered to be statistically significant. All statistical analyses were performed using SPSS 22.0 software (IBM Corp, Armonk, New York). 
Editorial

\section{Results}

The baseline characteristics of the patients of the two groups at diagnosis are listed in table 1.

\begin{tabular}{|c|c|c|c|}
\hline Characteristics & Group 1 (N=8) & Group 2 (N=9) & p-value \\
\hline Agein years [SD] & $9.4[2.9]$ & $10.8[3.6]$ & 0.395 \\
\hline Male sex & 5 & 6 & 1.0 \\
\hline H/O measles vaccination & 2 & 1 & 0.57 \\
\hline Low socioeconomic strata & 6 & 8 & 0.57 \\
\hline Mean [SD] latent period after measles infection (years) & $6.5[4.4]$ & $7.4[3.6]$ & 0.649 \\
\hline Jabbour stage 3-4 & 5 & 4 & 1.0 \\
\hline CSF pleocytosis (cells/mm $\left.{ }^{3}\right)$ & 4 & 4 & 1.0 \\
\hline Raised CSF protein (mg/dl) & 3 & 4 & 1.0 \\
\hline Mean CSF antibody titre [SD] (mIU/ml) & $2465.6[28.9]$ & $2476.4[20.8]$ & 0.38 \\
\hline MRI abnormality at admission & 6 & 5 & 0.61 \\
\hline Mean [SD] BAE scores at admission & $27.54[5.2]$ & $29.2[4.9]$ & 0.508 \\
\hline
\end{tabular}

Thus, table 1 showed no statistically significant difference in the baseline parameters of the patients of the 2 groups. MRI brain abnormalities were ill- defined high signal intensities in T2 weighted images, more commonly involving the posterior and periventricular white matter and less commonly the grey matter and the frontal areas. Patients with prolonged survival developed diffuse cerebral atrophy with hydrocephalus-ex-vacuo. Mean IgG antibody titre in CSF was estimated using enzyme immunoassay (EIA) technique. All patients were seronegative for HIV.

Table-2: Comparison of outcome among the 2 groups following treatment.

\begin{tabular}{|c|c|c|c|c|c|}
\hline Characteristics & $\begin{array}{c}\text { Group1 } \\
\mathbf{( N = 8 )}\end{array}$ & $\begin{array}{c}\text { Group2 } \\
\mathbf{( N = 9 )}\end{array}$ & OR (95\%CI) & RR (95\%CI) & p value \\
\hline Reduction in stage & 1 & 7 & $0.04(0.003-0.56)$ & $0.16(0.02-1.03)$ & 0.015 \\
\hline $\begin{array}{c}\text { Reduction in CSF antibody } \\
\text { titre }\end{array}$ & 0 & 5 & 0 & 0 & 0.029 \\
\hline $\begin{array}{c}\text { Mean CSF antibody titre } \\
\text { [SD] (mIU/ml) }\end{array}$ & $\begin{array}{c}2396.65 \\
{[89.5]}\end{array}$ & $\begin{array}{c}2301.94 \\
{[45.7]}\end{array}$ & & & 0.0135 \\
\hline Reduction in BAE score & 2 & 8 & $0.041(0.041-0.08)$ & $0.281(0.68-0.95)$ & 0.015 \\
\hline
\end{tabular}

Table 2 shows that treatment with intraventricular interferon alpha significantly improves the outcome of SSPE patients compared to those who had received oral ribavirin. Over the entire study period, 4 patients in Group 1 and 3 patient in group 2 died $(\mathrm{p}=0.63)$. The mean [SD] survival duration in years was 2.3 [1.0] in group 1 and 3.1 [0.4] in group 2 $(\mathrm{p}=0.042)$. All patients who expired were in Jabbour stages 3 or 4 at admission. Stage reduction occurred by only one grade for all patients.

Table-3: Analysis of factors affecting outcome of the patients.

\begin{tabular}{|c|c|c|c|c|c|c|}
\hline Characteristics & $\begin{array}{l}\text { Reduction in } \\
\text { stage }(\mathrm{N}=8)\end{array}$ & $\begin{array}{l}\text { No reduction } \\
\text { in stage } \\
(\mathrm{N}=9)\end{array}$ & $\begin{array}{c}\mathrm{p} \\
\text { value }\end{array}$ & $\begin{array}{l}\text { Reduced CSF } \\
\text { antibody titre } \\
\quad(\mathrm{N}=5)\end{array}$ & $\begin{array}{l}\text { No reduction in } \\
\text { CSF antibody } \\
\text { titre }(\mathrm{N}=12)\end{array}$ & $\begin{array}{c}\mathrm{p} \\
\text { value }\end{array}$ \\
\hline Mean age [SD] (years) & $10.2[2.6]$ & $6.7[3.3]$ & 0.029 & $9.8[5.2]$ & $5.9[2.5]$ & 0.049 \\
\hline $\begin{array}{l}\text { Mean [SD] latent } \\
\text { period after measles } \\
\text { infection (years) }\end{array}$ & $4.0[1.1]$ & $10[2.4]$ & 0.045 & $3.0[1.0]$ & $9[1.7]$ & 0.045 \\
\hline Jabbour stage 1-2 & 6 & 2 & & 3 & 2 & \\
\hline Jabbour stage 3-4 & 2 & 7 & 0.044 & 2 & 10 & 0.11 \\
\hline $\begin{array}{l}\text { Vaccinated children } \\
\qquad(\mathrm{N}=3)\end{array}$ & 2 & 1 & 0.57 & 2 & 1 & 0.19 \\
\hline
\end{tabular}


Editorial

Table 3 shows that longer latent period prior to the onset of SSPE symptoms after measles infection is associated with significantly higher chance of stage reduction and reduction in CSF anti-measels antibody titres. Lower Jabbour stage at presentation too significantly affects reduction in stage. Measels vaccination status did not affect outcome.

\section{Discussion}

This retrospective study demonstrated that intraventricular interferon alpha therapy is far superior to oral ribavirin, evidenced by significantly higher incidence of reduction in Jabbour stage, CSF antibody titre, and BAE scores and improved survival duration. Maimone [11] described the treatment of 3 patients of Stage II SSPE with different combinations of intrathecal and intravenous semipurified alpha-interferon, resulting in transient improvement of neurological symptoms and EEG in all, while cognitive function slightly improved in 2 of them. Clinical benefits gradually disappeared 2 to 6 months after cessation of IFN. Intrathecal antibody production did not change substantially.

Yazaki [12] described successful treatment of a 15 years old SSPE patient whose clinical condition had worsened (appearance of agnosia) despite being on oral isoprinosine. Generoso [13] in his international multicenter single blinded, randomized controlled trial of inosiplex alone (control group $=62$ patients) versus combined inosiplex and intra-ventricular interferon alpha (experimental group $=59$ patients) from 1996 until 2000 , did not demonstrate a clear superiority of one kind of treatment over another kind during the first 6 months after which the dropout rate was too high to complete any statistical analysis.

During the 2 years follow-up, no significant difference was noted on the BAE or the NDI (Neurological Disability Index)scores. Despite 121 subjects being randomized, only about half this number was used in the outcome evaluations primarily due to the lack of initial baseline assessments and also due to 19 protocol violations. Moodley [7] described a 19- years- old girl treated with intraventricular interferon alpha and showed significant improvement- EEG background returning to normal within 9 months, performing daily activities returning to school, being able to interact with her peers and learn new information within 30 months of treatment completion.

However, she had a relapse of SSPE and subsequently expired. Campbell [6] reported 6- years-old Filipino girl and 16- years-old Iraqi boy treated with intraventricular interferon alpha. The former died due to respiratory illness and the latter died due to respiratory distress syndrome and renal failure secondary to rhabdomyolysis. Campbell [6] also described a 14-yearold Canadian girl, treated initially with intravenous and later with intraventricular ribavirin, since CSF concentration of ribavirin were not adequate after intravenous injection to inhibit viral replication. The treatment did not produce any reversal in her clinical symptoms and signs and was hence, discontinued. In 1997, Anlar[14] compared the clinical outcome of 22 children treated with isoprinosine and intra-ventricular interferon alpha with 35 children treated with isoprinosine only. Interferon alpha was given as 100,000 units $/ \mathrm{m}^{2}$ increasing to 1 million units $/ \mathrm{m}^{2}$ per day for 5 days/week for six weeks and repeated at 2-6month intervals.

Little data was given for the control group and it was not clear why they did not receive interferon alpha given they were diagnosed during the same period. Half of the patients in the interferon alpha group improved initially and enjoyed a better survival profile until approximately eight years following diagnosis. Although many case reports describe clinical benefits for children treated with IFN, several others reportpoor outcomes $[15,16]$. CSF measles antibody titres fell with treatment in some cases; however, it was not always related to clinical improvement $[17,18,19]$. Nair [18] described the use of intrathecal and intravenous interferon alpha, the total dose varying from 15 million to 60 million units.

Clinical improvement as demonstrated by a decrease in scores on NDI occurred in three. Two of them relapsed five months after stopping the therapy but one patient maintained improvement two years after stopping treatment. There was no significant change in six patients. There were 3 deaths. A decrease in titres of antimissiles antibody in CSF was noted in two patients who showed clinical improvement, but in others there was no consistent correlation. No significant changes occurred in EEG during the treatment period.

Thus they opined that use of intravenous combined with intrathecal interferon is not promising in SSPE. Hosoya [20] described treatment of 5 patients with intraventricular ribavirin with variable results. 3 patients had reduction in the CSF measles antibody titre, reduction in Jabbour staging, improvement of NDI scores, stoppage of myoclonus and periodic synchronous discharge on EEG disappeared. However, 2 patients had neither clinical improvement nor a decrease in the measles antibody titer in the CSF. There were mild and 
Editorial

transient side effects with intraventricular ribavirin, such as lip and gingival swelling, conjunctival hyperemia, headache, and drowsiness. Anemia, which is commonly encountered with systemic ribavirin administration, was not observed with intraventricular ribavirin.

In our study, higher chance of reduction of Jabbour staging and CSF antibody titre is significantly determined by higher mean age of onset of SSPE, higher mean latent period between measles infection and onset of SSPE symptoms and lower Jabbour stages at presentation. However, Malik [21] described that unvaccinated patients had more fulminant course than immunized patients. Among patients with no history of measles in the past, males showed significantly higher incidence of subacute course of the disease than in females $(64.7 \%$ vs. $16.7 \%)$. Sex, malnutrition, age at contracting measles infection, and age of onset of clinical manifestations of SSPE had no effect on the clinical course. But patients with shorter latent duration between the age of contracting measles infection and onset of SSPE symptoms had a trend of having more rapid course.

\section{Conclusion}

Thus, no curative therapy is currently available for SSPE [1,7]. Hence, based on our experience in this study, we recommend the following-1) patients of SSPE should be treated with interferon alpha 2 b, whenever possible, either as monotherapy or in combination with other drugs.

2) Intraventricular administration is the preferred mode, since it provides the highest central nervous system concentration of the drug. 3) Sodium valproate is the preferred anticonvulsant for SSPE patients, with addition of levetiracetam and zonisamide sequentially upon refractoriness of the seizures.

4) Future multicenter international clinical comparative trials should be conducted in developing countries in which SSPE is still endemic to investigate the effectiveness of different combination treatments of immunomodulators and antiviral drugs with larger number of subjects.

\section{What is already known?}

SSPE is a progressive neuro degenerative disease caused by proliferating defective measles virus, without any curative therapy at present; patients facing grim prognosis despite multiple courses of mono/polytherapy of different combinations via different routes.

\section{What this study adds?}

Intraventricular interferon alpha $2 \mathrm{~b}$ is an effective monotherapy for SSPE resulting in clinical down staging and improved survival.

Funding: Nil, Conflict of interest: None initiated, Perission from IRB: Yes

Author contribution- Dr. Kartick Chandra Ghosh, Dr. Ramesh Bhattacharya and Dr. Gourango Prosad Mondal provided intellectual inputs and planned the treatment protocols. Dr. Saikat Ghosh, Dr. Manoj Mahata, and Dr. Sarbajit Das performed the day to day management of the patients. Dr Suman Das wrote the manuscript.

\section{References}

1. Gutierrez J, Issacson RS, Koppe BS. Subacute sclerosing panencephalitis: an update. Developmental Medicine \& Child Neurology 2010;52(10): 901-907.

2. Saha V, John TJ, Mukundan P, et al. High incidence of subacute sclerosing panencephalitis in south India. Epidemiol Infect. 1990 Feb;104(1):151-6

3. Jagtap SA, Nair MD, Kambale HJ. Subacute sclerosing panencephalitis: A clinical appraisal. Ann Indian Acad Neurol. 2013;16(4):631-633.

4. Garg Rk. Subacute sclerosing panencephalitis. Postgrad Med J. 2002 Feb;78(916):63-70

5. Aaby P, Bukh J, Lisse IM, et al. Risk factors in subacute sclerosing panencephalitis: age- and sexdependent host reactions or intensive exposure? Rev Infect Dis. 1984 Mar-Apr;6(2):239-50.

6. Campbell C, Levin S, Humphreys P, Walop W, Brannan R. Subacute Sclerosing Panencephalitis: Results of the Canadian Paediatric Surveillance Program and review of the literature. BMC Pediatr. 2005; 5 (12):47.DOI:10.1186/1471-2431-5-47.

7. Moodley K, Bill PLA, Patel VB. Interferon alpha in subacute sclerosing panencephalitis: Case report and review of the literature. Southern African Journal of Infectious Diseases 2016;31(4):130-134.

8. Dyken P, DuRant R, Shmunes P. Subacute sclerosing panencephalitis surveillance- United States. MMWR. 1982; 31 (43):585-588.

9. Gascon G, Yamani S, Crowell J, et al. A Combined oral isoprinosine- intraventricular alpha-interferon 


\section{Editorial}

therapy for subacute sclerosing panencephalitis. Brain Dev. 1993 Sep-Oct;15(5):346-55.

10. Nester MJ. Use of a brief assessment examination in a study of subacute sclerosing panencephalitis. J Child Neurol. 1996;11(3):173-180.DOI:10.1177/088307389 601100301.

11. Maimone $\mathrm{D}$, Grimaldi LM, Incorpora $\mathrm{G}$, et al. Intrathecal interferon in subacute sclerosing panencephalitis. Acta Neurol Scand. 1988 Sep;78(3): 161-6.

12. Yazaki M, Yamazaki M, Urasawa N, et al. Successful treatment with alpha-interferon of a patient with chronic measles infection of the brain and parkinsonism. Eur Neurol. 2000;44(3):184-6.

13. Generoso G, Gascon GG Randomized treatment study of inosiplex versus combined inosiplex and intraventricular interferon-alpha in subacute sclerosing panencephalitis (SSPE): international multicenter study. J Child Neurol 2003;18(12):819-827.

14. Anlar B, Yalaz K, OktemF, et al. Long-term followup of patients with subacute sclerosing panencephalitis treated with intraventricular alpha-interferon.

Neurology. 1997 Feb;48(2):526-8.

15. Fayad NM, Yamout BI, Mroueh S. Alpha-interferon in the treatment of subacute sclerosing panencephalitis. J Child Neurol. 1997;12(8):486-488.
16. Solomon T, Hart CA, Vinjamuri S, et al. Treatment of subacute sclerosing panencephalitis with interferonalpha, ribavirin, and inosiplex. J Child Neurol. 2002 Sep; 17 (9):703-5.

17. Panitch HS, Gomez-Plascencia J, Norris FH, Cantell K, Smith RA. Subacute sclerosing panencephalitis: remission after treatment with intravenous interferon. Neurology. 1986;36(4):562-566.

18.Nair K, Swamy HS,Arunodaya GR, et al Intravenous with intrathecal interferon in subacute sclerosing panencephalitis. Neurol India. 1995 Jan-Mar; 43 (1): 20-25.

19. Yalaz K, Anlar B, Oktem F, et al. Intraventricular interferon and oral inosiplex in the treatment of subacute sclerosing panencephalitis. Neurology. 1992 Mar; 42 (3 Pt 1):488-91.

20. Hosoya M, Mori S, Tomoda A, et al. Pharmacokinetics and effects of ribavirin following intraventricular administration for treatmentof subacute sclerosing panencephalitis. Antimicrob Agents Chemother. 2004 Dec;48(12):4631-5

21. Malik M, Saeed M, Qureshi AU, Ahmed N, Akram M. Predictors of Clinical Course of Subacute Sclerosing Panencephalitis: Experience at the Children's Hospital, Lahore. Journal of the College of Physicians and Surgeons Pakistan 2010;20(10):671-674.

\section{How to cite this article?}

Ghosh K.C, Bhattacharya R, Ghosh S, Mahata M, Das S, Das S, Mondal G.P. Comparative study of the outcome of pediatric subacute sclerosing pan-encephalitis patients treated with oral ribavirin versus intraventricular interferon alpha 2b via Ommaya reservoir. Int J Pediatr Res. 2018;5(6):320-325.doi:10.17511/ijpr.2018.i06.04. 